\title{
Agronomic Evaluation of New Varieties of Cassava (Manibot esculenta Crantz) under Different Rates and Modes of NPK (12-12-17-2) Fertilizer Application in Two Seasons
}

\author{
Stella O. MUOJIAMA*, Christian U. AGBO, Simon C. EZE, \\ Charles U. UBA \\ University of Nigeria, Faculty of Agriculture, Department of Crop Science, Nsukka, Enugu State, Nigeria; \\ stellypotential@yahoo.com (*correspondingauthor); christian.agbo@unn.edu.ng; simon.eze@unn.edu.ng; charlesuba192@gmail.com
}

\begin{abstract}
A study was carried out at the Teaching and Research Farm of the Department of Crop Science, University of Nigeria, Nsukka to evaluate the growth and yield of four improved cassava varieties, determine their optimum NPK fertilizer rate and the best modes of fertilizer application for increased productivity. The experiment was factorial laid out in a randomized complete block design with three replications. Four varieties of cassava, four fertilizer rates and three modes of fertilizer application were used for the study. The early planting had higher survival count, number of leaves, tuber and garri yield when compared with late planting. The variety TMS 980505 gave significantly $(\mathrm{p}<0.05)$ higher number of leaves, tuber and garri yields of 39.8 and $9.68 \mathrm{t} / \mathrm{ha}$, respectively, at 12 months. At 6 months of crop growth, $200 \mathrm{~kg} / \mathrm{ha}$ fertilizer application rate gave significantly higher tuber and garri yield of $24.69 \mathrm{t} / \mathrm{ha}$ and $5.15 \mathrm{t} / \mathrm{ha}$, respectively. Furthermore, $400 \mathrm{~kg} / \mathrm{ha}$ of NPK fertilizer rate gave higher tuber and garri yields of 39.4 and $10.12 \mathrm{t} / \mathrm{ha}$, respectively at 12 months of crop growth. Split application of fertilizer gave significantly higher tuber and garri yield from single application, though it is statistically similar to split-split application. Therefore, early planting, $400 \mathrm{~kg} / \mathrm{ha}$ fertilizer rate and split application should be adopted for cassava production. The variety TMS 980505 with higher growth and yield should also be adopted by farmers for cassava production in Nsukka derived savannah agro-ecology.
\end{abstract}

Keywords: Cassava production; evaluation; fertilizer application; season; yield

\section{Introduction}

Cassava (Manihot esculenta Crantz.) is a perennial shrub of the family Euphorbiaceae. It is a root crop that is propagated vegetatively from stem cuttings for commercial purposes, but can also be propagated by seed. Due to its adaptability to marginal soils and erratic rainfall, high productivity per unit of land and labour, the possibility of supply throughout the year has been obtained (Nweke et al., 2002). The adaptation to different edapho-climatic conditions (Adeniji et al., 2011) makes cassava a favorite dry season crop grown in inland valleys in West and central Africa (Lahai and Ekanayake, 2009), noting it is highly susceptible to excessive water (Ande, 2011).

Cassava tubers contain about $92.2 \%$ carbohydrates and $3.2 \%$ protein in its dry matter, thus is said to have high energy content. It leaves and tender shoots are important source of vitamins, minerals and proteins (Balagopalan,
2002; Nweke et al., 2002). The tubers are mostly processed into Cassava flour, garri and fufu in Nigeria. It can also be cooked or eaten pounded and consumed in its raw form, most especially the sweet variety (Ogundari and Ojo, 2007). Cassava products are used in various forms for human consumption, livestock feed and manufacturing of industrial products (Ene, 1992). According to IITA (1990), cassava products are also important feed stuff for livestock formulation. For example, it has a capacity of substituting up to $44 \%$ maize in pig feed without any reduction in the performance (Okeke, 1998); it can also be observed that in compounding feed for pigs, broiler, pullets and layers, cassava meal plays a significant role.

To increase the yield potential of cassava, the crop has been reported to respond to good soil fertility and adequate fertilizer (Howeler, 1996). According to Howeler (1991) the major nutrients required by cassava for optimum top growth and tuber yields are nitrogen $(\mathrm{N})$ and potassium (K). Soils that have low $\mathrm{N}(<0.10 \%$ total $\mathrm{N})$ and $\mathrm{K}(<0.15$ 
108

$\mathrm{meg} / 100 \mathrm{~g}$ ) will require an additional fertilizer for optimum tuber yield (Kang and Okeke, 1991). Adequate K levels in the soil stimulate the response to $\mathrm{N}$ fertilizers, but excess amount of both nutrient leads to luxuriant growth at the expense of tuber formation (Onwueme and Charles 1994). Moreso, Kang and Okeke (1991) reported that cropping systems influence fertilizer requirements of cassava; for example, the continuous cropping of cassava leads to fast depletion of major nutrients, especially $\mathrm{N}$ and $\mathrm{K}$ and will require fertilizer supplement to give stable yield. Cassava removes about $55 \mathrm{~kg} / \mathrm{ha} \mathrm{N}, 132 \mathrm{~kg} / \mathrm{ha} \mathrm{P}$ and $112 \mathrm{~kg} / \mathrm{ha} \mathrm{K}$ (Howeler, 1991). Sittibusaya and Kurmarohita (1978) reported that after 15 years of continuous cassava production without fertilization in South East Thailand, yields dropped from an initial level of $30 \mathrm{t} / \mathrm{ha}$ to only $17 \mathrm{t} / \mathrm{ha}$. When these were exhausted, soils were fertilized with $164 \mathrm{~kg}$ and 312 $\mathrm{kg} / \mathrm{ha}$, yield increased from 22 to $41 \mathrm{t} / \mathrm{ha}$.

Furthermore, in recent years the production of cassava has increased, but its current production rate per hectare (8$10 \mathrm{t} / \mathrm{ha}$ ) is far lower than the potential (30-60 t/ha). However, causes of low productivity in cassava production are the high cost of farm inputs such as fertilizer and planting materials, deficiencies in the supply and delivery of farm inputs, soil fertility status of the farm land, cropping system adopted, the rainfall pattern during the growing season, sector dominance (90\%) by small holder, resourcepoor farmers etc. The indifference towards low productivity can also be attributed to the low and unstable prices of cassava tubers. Available record shows that both in absolute terms and on per hectare basis, Nigeria ranks among the lowest consumers of fertilizers in the world (Aderi et al., 2010). Its current fertilizer use is about one million tonnes per annum, while the projected demand estimate is 3.7 million tonnes (Aba, 2010). Average worldwide rates are 93 $\mathrm{kg} / \mathrm{ha}$ of NPK, while the rate for Nigeria is about $13 \mathrm{~kg} / \mathrm{ha}$ (Aderi et al., 2010).

Hence, it is important to determine NPK fertilizer requirements and the best mode of application for increased cassava productivity, in improved cassava varieties. Therefore, the objectives of the present study were to evaluate growth and yield of four improved cassava varieties, to determine the optimum NPK fertilizer rate and the best mode of fertilizer application for increased productivity.

\section{Materials and Methods}

The field trail was conducted at the Teaching and Research farm of the Department of Crop Science, University of Nigeria, Nsukka during 2014 planting season. Nsukka derived as savannah agro-ecology are and is located at latitude $06^{\circ} 54^{\circ} \mathrm{N}$, longitude $07^{\circ} 24 \mathrm{E}$ and altitude of 550 $\mathrm{m}$ above sea level. The rainfall is bimodal with the peaks in June and September, respectively. The soil is broadly characterized as sandy clay loam ultisol (oxic paleustult) and belongs to Nkpologu series (Mbagwu, 1992). The cassava varieties were planted at two seasons (e.g. early and late season). During the early season, cassava was planted between April to May at the stabilization of rainy season, while during the late season, it was planted between July to August at the mid - month of rainy season, which are the planting seasons of cassava in this agro-ecology. The experiment was $4 \times 4 \times 3$ factorial laid out in randomized complete block design (RCBD) with 3 replications. The factors were four varieties of cassava (TMS 011368 (yellow root), TME 419, TMS 980505 and TMS 05 10), four rates or levels of NPK fertilizer (0 kg/ha, $200 \mathrm{~kg} / \mathrm{ha}, 400 \mathrm{~kg} / \mathrm{ha}$ and $600 \mathrm{~kg} / \mathrm{ha}$ ) and three modes of fertilizer application (single at 4 weeks after planting, split at 4 and 8 weeks after planting and split-split at 4,8 and 12 weeks after planting). The treatments comprised all possible combinations of cassava varieties, fertilizer rates and modes of fertilizer application. The experimental field measuring $2,461 \mathrm{~m}^{2}$ was cleared, ploughed, harrowed, ridged and marked out into three blocks. Each block was sub-divided into 48 plots, each with a dimension of $5 \mathrm{~m} \times 2 \mathrm{~m}$ per plot. The cassava was planted at a spacing of $1 \mathrm{~m} \times 1 \mathrm{~m}$ to obtain a plant population of $10,000 \mathrm{~m}^{2}$ per hectare. Healthy cassava stem collected from National Root Crops Research Institute (NRCRI), Umudike, were cut between 4-6 nodes ,where two nodes were exposed above the soil surface when planted. The fertilizer used was NPK-Mg 12-12-17-2 and was applied by band placement method. Weeding was done three times manually during the period of the research.

Soil samples were randomly collected before planting from three representative locations by augering to the depth of $20 \mathrm{~cm}$ with a steel auger. The samples were bulked together and the composite samples were taken for laboratory analysis to determine the physical and chemical characteristics of the site. The organic matter was determined using the Walkey \& Black method (Nelson and Sommers, 1996). Particle size analysis was done by pipette method (Gee and Bauders, 1986); soil $\mathrm{pH}$ in water was determined using soil: water ratio of $1: 2$ by a $\mathrm{pH}$ meter with a glass electrode. Exchangeable bases in the samples were extracted in $0.1 \mathrm{M} \mathrm{NH}_{4} 0 \mathrm{AC}$ at $\mathrm{pH}$ 7.0. Calcium $(\mathrm{Ca})$ and Magnesium $(\mathrm{Mg})$ in the extract, after which were read by Atomic Absorption Spectrophotometer. Total Nitrogen $(\mathrm{N})$ in the soil was determined by Kjedahl digestion (Bremner, 1996). Sodium $(\mathrm{Na})$ and Potassium $(\mathrm{K})$ were analyzed by using flame photometry. Exchangeable acidity was determined by extracting with $1 \mathrm{M} \mathrm{KCl}$ and determined by $\mathrm{NaOH}$ titration (Sims, 1996). Available phosphorous was determined by Bray-1 extraction and determined colourimetrically by the molybdenum blue procedure (Bray and Kurtz, 1945).

The following data were collected on the agronomic and processed parameters on each plot: survival count, number of branches, number of leaves, plant height, stem girth, canopy diameter, tuber and garri yields. After 3 weeks of planting, the surviving sprouted stems were counted to ascertain the plot population. Numbers of branches were visually counted on 2 and 4 months after planting (MAP) to note the architecture and branching types. Number of leaves was visually counted on 2 and MAP. Stem girth was measured at 6, 8, 10 and 12 MAP when the stem was of good size and was measured in millimetre $(\mathrm{mm})$ using vernier calipers. Canopy diameter was taken at 8,10 and 12 MAP to get the area covered by the leaves in centimetre $(\mathrm{cm})$. Tuber yield measurement was taken in weight at three different sampling periods e.g. at 6 months, 9 months and 12 months after planting (MAP).

\section{Statistical analysis}

The data collected were analyzed according to the procedure outlined by Gomez and Gomez (1984) for a factorial experiment laid out in a randomized complete block design. 


\section{Results}

Meteorological data of the experimental site

The meteorological data showed that bimodal peaks of rain in 2014 were in June $(271.79 \mathrm{~mm})$ and September $(401.99 \mathrm{~mm})$ (Table 1). The amount of rain in August and December of 2014 were low. There was no rain in January, 2015 and the subsequent months of February to April were low (34-56 $\mathrm{mm}$ in a month).

\section{Physico-chemical properties of the soil of the experimental site \\ Physico-chemical properties of the experimental sites before planting showed that the textural class of the site of the early season planting was loamy sand, while the site of the late season planting was sandy loam (Table 2). The soil of the experimental sites was highly acidic with $\mathrm{pH}$ in $\mathrm{H}_{2} \mathrm{O}$ and KCL of 4.7 and 3.8 for the early planting and 4.8 and 3.7 for the late season planting, respectively. The soils of both seasons were characterized to be low in organic matter, exchangeable bases and cation exchange capacity (CEC).}

Agronomic and yield performance of the varieties at early season planting

However, under early establishment TME 419 variety gave significantly $(\mathrm{p}<0.05)$ higher percentage survival count of $91 \%$ although it was statistically similar to TMS 011368 (yellow root) with 90.8\% (Table 3). The variety TMS 9805 05 gave significantly $(p<0.05)$ higher number of leaves, while TME 419 variety had significantly $(\mathrm{p}<0.05)$ lower number of leaves in the second and fourth month of planting. Fertilizer application rate of $200 \mathrm{~kg} / \mathrm{ha}$ gave significantly higher number of leaves at the second month after planting, while $600 \mathrm{~kg} /$ ha gave significantly $(\mathrm{p}<0.05)$ higher number of leaves in the fourth month. However, the control $(0 \mathrm{~kg} / \mathrm{ha})$ gave the lowest number of leaves in both months. Single application of fertilizer gave significantly $(\mathrm{p}<0.05)$ higher number of leaves at fourth month of crop growth. The variety TMS 980505 gave the highest number of branches at two months after planting (MAP) and it was significantly $(\mathrm{p}<$ 0.05 ) higher than other varieties. The rate and mode of fertilizer application did not cause any significant differences in the number of branches of the cassava varieties over the four months after planting in the early season planting.

Furthermore, the variety TMS $98 \quad 05 \quad 05$ gave significantly higher tuber and garri yields of 39.8 and 9.68 $\mathrm{t} / \mathrm{h}$ a, respectively at twelfth months of crop growth, although it was statistically similar to TMS 0105 (Table 5). The rate of $200 \mathrm{~kg} / \mathrm{ha}$ of NPK gave significantly $(\mathrm{p}<0.05)$ higher tuber and garri yields of $24.69 \mathrm{t} / \mathrm{ha}$ and $5.15 \mathrm{t} / \mathrm{ha}$, respectively at 6 months of growth. However, the rate of $400 \mathrm{~kg} / \mathrm{ha}$ of NPK gave significantly $(\mathrm{p}<0.05)$ higher tuber and garri yields of 39.4 and 10.12 t/ha at 12 months of growth. Split-split application of NPK fertilizer gave the highest yield of tuber and garri at 12 months of growth. Significantly higher peel weight of $8.94 \mathrm{t} /$ ha was obtained in TME 419 at $6 \mathrm{MAP}$, as well as non-significant higher peel weight of $6.25 \mathrm{t} / \mathrm{ha}$ at 9 MAP. At 12 MAP, TMS 980505 gave significantly higher peel weight of $9.36 \mathrm{t} / \mathrm{ha}$. More so, TMS 980505 showed higher canopy diameter and stem girth throughout the months of growth (Table 4). TMS 011368 showed non significant lower canopy diameter from 8 to 12 months after planting. The $400 \mathrm{~kg} / \mathrm{ha}$ rate of NPK fertilizer gave higher canopy diameter and stem girth at $12 \mathrm{MAP}$.

Table 1. Meteorological data for the cropping seasons of 2014 and 2015

\begin{tabular}{|c|c|c|c|c|c|}
\hline \multirow{2}{*}{ Month } & \multirow{2}{*}{ Rainfall (mm) } & \multicolumn{2}{|c|}{ Temperature $\left({ }^{\circ} \mathrm{C}\right)$} & \multicolumn{2}{|c|}{ Relative Humidity (\%) } \\
\hline & & Min & Max & $10 \mathrm{am}$ & $4 \mathrm{pm}$ \\
\hline \multicolumn{6}{|l|}{ Year 2014} \\
\hline April & 105.16 & 22.30 & 31.30 & 69.93 & 70.53 \\
\hline May & 241.14 & 21.06 & 28.29 & 72.26 & 72.26 \\
\hline June & 271.79 & 20.87 & 29.13 & 72.00 & 72.00 \\
\hline July & 195.81 & 20.90 & 27.74 & 72.19 & 72.19 \\
\hline August & 92.36 & 20.71 & 27.29 & 73.00 & 73.00 \\
\hline September & 401.99 & 20.33 & 27.90 & 73.00 & 73.00 \\
\hline October & 211.08 & 20.84 & 28.90 & 73.00 & 72.77 \\
\hline November & 77.22 & 21.00 & 30.07 & 73.80 & 71.97 \\
\hline December & 4.83 & 19.03 & 30.65 & 70.58 & 70.06 \\
\hline Total & 1601.38 & 187.04 & 261.27 & 649.70 & 647.78 \\
\hline Mean & 177.93 & 20.78 & 29.03 & 72.20 & 71.98 \\
\hline \multicolumn{6}{|l|}{ Year 2015} \\
\hline January & Nil & 20.52 & 30.32 & 61.42 & 59.58 \\
\hline February & 56.64 & 22.68 & 32.04 & 70.11 & 64.21 \\
\hline March & 34.8 & 22.61 & 32.29 & 70.61 & 70.19 \\
\hline April & 39.63 & 22.4 & 31.47 & 71.03 & 67.67 \\
\hline May & 267.98 & 21.81 & 30.71 & 71.65 & 71.42 \\
\hline June & 121.43 & 21.17 & 29.07 & 76 & 76 \\
\hline July & 110.49 & 20.61 & 27.87 & 76 & 76.03 \\
\hline August & 410.4 & 20.43 & 27.69 & 76 & 76.1 \\
\hline Total & 630.97 & 151.8 & 213.77 & 496.82 & 485.1 \\
\hline Mean & 90.14 & 21.69 & 30.54 & 70.97 & 69.3 \\
\hline
\end{tabular}


110

Table 2. Physical and chemical properties of the soil in the experiment area

\begin{tabular}{|c|c|c|}
\hline Properties & Early season site & Late season site \\
\hline \multicolumn{3}{|l|}{ Particle size distribution (\%) } \\
\hline Clay & 10 & 14 \\
\hline Silt & 5 & 7 \\
\hline Fine Sand & 33 & 43 \\
\hline Coarse Sand & 52 & 36 \\
\hline Textural Class & Loamy Sandy & Sandy Loam \\
\hline \multicolumn{3}{|l|}{ Chemical Properties } \\
\hline $\mathrm{pH}\left(\mathrm{H}_{2} \mathrm{O}\right)$ & 4.7 & 4.8 \\
\hline $\mathrm{pH}(\mathrm{KCL}))$ & 3.8 & 3.7 \\
\hline Organic Carbon (\%) & 0.95 & 1.25 \\
\hline Organic Matter (\%) & 1.63 & 2.15 \\
\hline Total nitrogen (\%) & 0.084 & 0.098 \\
\hline \multicolumn{3}{|l|}{ Exchange bases (Meq/100 g soil) } \\
\hline Sodium $(\mathrm{Na}+)$ & 0.12 & 0.15 \\
\hline Potassium $(\mathrm{K}+)$ & 0.15 & 0.19 \\
\hline Calcium $(\mathrm{Ca}+)$ & 11.4 & 13.2 \\
\hline Magnesium $(\mathrm{Mg}+)$ & 0.4 & 2.4 \\
\hline Cation Exchange Capability (Meq/100 g soil) & 13 & 16.4 \\
\hline Base Saturation (\%) & 92.85 & 97.2 \\
\hline Phosphorus (ppm) & 6.53 & 3.73 \\
\hline \multicolumn{3}{|l|}{ Exchangeable Acidity (Meq/100 g soil) } \\
\hline Aluminium Oxide (AL+) & - & - \\
\hline Hydrogen Oxide (H+) & 2.4 & 2.4 \\
\hline - $\quad=$ Not detected & & \\
\hline
\end{tabular}

Table 3. Survival count (\%) and number of leaves and branches of the early season planted cassava varieties as influenced by rates and modes of fertilizer application at four months after planting (MAP)

\begin{tabular}{|c|c|c|c|c|c|}
\hline \multirow{3}{*}{$\begin{array}{c}\text { Treatments } \\
\text { Varieties }\end{array}$} & Survival count $(\%)$ & \multicolumn{2}{|c|}{ Number of leaves/Plant } & \multicolumn{2}{|c|}{ Number of branches/Plant } \\
\hline & \multicolumn{5}{|c|}{ Months after planting } \\
\hline & & 2 & 4 & 2 & 4 \\
\hline \multicolumn{6}{|c|}{ Early season } \\
\hline TMS 0I 1368 & 90.8 & 10.55 & 71.80 & 1.84 & 3.54 \\
\hline TME 419 & 91.1 & 10.05 & 38.10 & 1.73 & 3.09 \\
\hline TMS 980505 & 80.6 & 14.78 & 84.90 & 2.18 & 3.64 \\
\hline TMS 0510 & 84.2 & 11.78 & 44.30 & 1.79 & 2.92 \\
\hline $\operatorname{LSD}(\mathrm{p}<0.05)$ & 4.5 & 0.85 & 6.01 & 0.26 & NS \\
\hline \multicolumn{6}{|l|}{ Rates } \\
\hline 0 & & 10.27 & 54.20 & 1.77 & 3.33 \\
\hline 200 & & 12.54 & 56.90 & 1.98 & 2.87 \\
\hline 400 & & 12.17 & 61.70 & 1.91 & 3.61 \\
\hline 600 & & 10.47 & 65.30 & 1.87 & 3.38 \\
\hline $\operatorname{LSD}(\mathrm{p}<0.05)$ & & 0.85 & 6.01 & NS & NS \\
\hline \multicolumn{6}{|l|}{ Modes } \\
\hline Single & & 11.40 & 67.30 & 1.91 & 3.37 \\
\hline Split & & 11.85 & 54.60 & 1.90 & 3.10 \\
\hline Split-Split & & 12.12 & 57.40 & 1.84 & 3.42 \\
\hline $\operatorname{LSD}(\mathrm{p}<0.05)$ & & NS & 5.21 & NS & NS \\
\hline \multicolumn{6}{|c|}{ Late season } \\
\hline TMS 0I 1368 & 62.8 & 8.55 & 61.80 & 1.44 & 3.24 \\
\hline TME 419 & 61.1 & 7.05 & 28.40 & 1.35 & 3.09 \\
\hline TMS 980505 & 70.6 & 12.58 & 74.60 & 2.00 & 3.33 \\
\hline TMS 0510 & 64.2 & 8.80 & 34.60 & 1.35 & 2.22 \\
\hline $\operatorname{LSD}(\mathrm{p}<0.05)$ & 1.5 & 0.85 & 5.01 & 0.26 & NS \\
\hline \multicolumn{6}{|l|}{ Rates } \\
\hline 0 & & 8.25 & 45.20 & 1.12 & 3.00 \\
\hline 200 & & 10.52 & 48.90 & 1.00 & 2.11 \\
\hline 400 & & 10.14 & 52.70 & 1.00 & 3.21 \\
\hline 600 & & 9.45 & 55.30 & 1.31 & 3.08 \\
\hline $\operatorname{LSD}(p<0.05)$ & & 0.85 & 6.01 & NS & NS \\
\hline \multicolumn{6}{|l|}{ Modes } \\
\hline Single & & 10.40 & 50.30 & 1.01 & 3.16 \\
\hline Split & & 9.85 & 49.30 & 0.98 & 3.10 \\
\hline Split-Split & & 9.12 & 47.40 & 1.04 & 3.02 \\
\hline $\operatorname{LSD}(\mathrm{p}<0.05)$ & & NS & NS & NS & NS \\
\hline
\end{tabular}


Table 4. Influence of cassava varieties $\mathrm{x}$ rates $\mathrm{x}$ modes of fertilizer application on their canopy diameter and stem girth at twelve months after planting (MAP) of the early season planting

\begin{tabular}{|c|c|c|c|c|c|c|c|}
\hline \multirow{3}{*}{$\begin{array}{c}\text { Treatments } \\
\text { Varieties }\end{array}$} & \multicolumn{3}{|c|}{ Canopy diameter $(\mathrm{cm})$} & \multicolumn{4}{|c|}{ Stem girth $(\mathrm{mm})$} \\
\hline & \multicolumn{7}{|c|}{ Months after planting } \\
\hline & 8 & 10 & 12 & 6 & 8 & 10 & 12 \\
\hline \multicolumn{8}{|c|}{ Early season } \\
\hline TMS 0I 1368 & 96.70 & 101.50 & 103.20 & 3.65 & 4.01 & 4.55 & 5.66 \\
\hline TME 419 & 113.60 & 114.70 & 116.00 & 4.01 & 4.48 & 4.56 & 6.07 \\
\hline TMS 980505 & 112.70 & 115.40 & 121.70 & 4.35 & 4.97 & 5.06 & 5.78 \\
\hline TMS 0510 & 100.00 & 101.0 & 105.70 & 4.12 & 4.37 & 4.39 & 6.00 \\
\hline $\operatorname{LSD}(\mathrm{p}<0.05)$ & NS & 11.39 & 15.06 & 0.43 & 0.53 & NS & NS \\
\hline \multicolumn{8}{|l|}{ Rates } \\
\hline 0 & 101.90 & 104.50 & 107.70 & 4.22 & 4.52 & 4.56 & 5.62 \\
\hline 200 & 101.30 & 103.80 & 105.40 & 4.26 & 4.54 & 4.45 & 5.42 \\
\hline 400 & 115.90 & 117.00 & 120.20 & 4.23 & 4.48 & 4.92 & 6.31 \\
\hline 600 & 108.80 & 110.20 & 114.20 & 3.72 & 4.29 & 4.63 & 6.12 \\
\hline $\operatorname{LSD}(\mathrm{p}<0.05)$ & NS & NS & NS & NS & NS & NS & NS \\
\hline \multicolumn{8}{|l|}{ Modes } \\
\hline Single & 109.90 & 110.20 & 110.90 & 4.32 & 4.51 & 4.54 & 5.95 \\
\hline Split & 105.60 & 105.80 & 107.50 & 4.25 & 4.59 & 5.06 & 6.06 \\
\hline Split-Split & 104.50 & 105.20 & 111.30 & 4.01 & 4.27 & 4.33 & 5.62 \\
\hline LSD $(p<0.05)$ & NS & NS & NS & NS & NS & NS & NS \\
\hline \multicolumn{8}{|c|}{ Late season } \\
\hline TMS 0I 1368 & 60.40 & 71.50 & 73.20 & 2.85 & 3.01 & 3.53 & 3.66 \\
\hline TME 419 & 73.60 & 72.70 & 76.00 & 3.01 & 3.48 & 3.56 & 3.67 \\
\hline TMS 980505 & 73.70 & 75.40 & 78.70 & 3.35 & 3.67 & 3.76 & 3.78 \\
\hline TMS 0510 & 60.00 & 71.20 & 75.70 & 3.12 & 3.27 & 3.39 & 3.40 \\
\hline $\operatorname{LSD}(\mathrm{p}<0.05)$ & NS & NS & NS & NS & NS & NS & NS \\
\hline \multicolumn{8}{|l|}{ Rates } \\
\hline 0 & 60.60 & 61.50 & 62.70 & 3.02 & 3.12 & 3.56 & 3.62 \\
\hline 200 & 71.30 & 73.80 & 75.40 & 3.26 & 3.54 & 3.45 & 3.42 \\
\hline 400 & 72.40 & 81.00 & 85.20 & 3.23 & 3.48 & 3.92 & 3.91 \\
\hline 600 & 72.80 & 80.20 & 84.20 & 3.52 & 3.29 & 3.63 & 3.62 \\
\hline $\operatorname{LSD}(\mathrm{p}<0.05)$ & NS & NS & NS & NS & NS & NS & NS \\
\hline \multicolumn{8}{|l|}{ Modes } \\
\hline Single & 89.20 & 80.20 & 81.90 & 3.12 & 3.51 & 3.42 & 3.55 \\
\hline Split & 75.60 & 75.80 & 85.50 & 3.25 & 3.49 & 3.60 & 3.26 \\
\hline Split-Split & 74.50 & 75.20 & 86.30 & 3.01 & 3.27 & 3.33 & 3.62 \\
\hline LSD $(p<0.05)$ & NS & NS & NS & NS & NS & NS & NS \\
\hline
\end{tabular}

Agronomic and yield performance of the varieties at late season planting

In the late season planting, variety TMS 980505 gave significantly higher survival count of $70.6 \%$ (Table 4). The survival count in the later season was generally reduced when compared with the earlier planting. The variety TMS 980505 also gave significantly higher number of leaves and branches at the late season planting. Fertilizer application rate of $200 \mathrm{~kg} / \mathrm{ha}$ and $600 \mathrm{~kg} / \mathrm{ha}$ gave significantly higher number of leaves at 2 and $4 \mathrm{MAP}$, respectively. The TMS 980505 variety gave significantly $(\mathrm{p}<0.05)$ higher canopy diameter at the 10 and 12 MAP (Table 4). The variety also gave significantly higher stem girth at the 6 and 8 MAP. The fertilizer rate of $400 \mathrm{~kg} / \mathrm{ha}$ gave non-significant higher canopy diameter at 8,10 and $12 \mathrm{MAP}$. Single dose of fertilizer gave non-significant higher stem girth and canopy diameter at 6 and $8 \mathrm{MAP}$, respectively. There were nonsignificant effect of the rates and modes of fertilizer application on the canopy diameter and stem girth of the varieties over the months of the growth.

The variety TMS 980505 gave significantly $(\mathrm{p}<0.05)$ higher tuber and garri yields at the sixth month of test period of the late planting season (Table 5). The variety also had higher non-significant yield at the twelfth months of harvest. The TME 419 variety gave higher garri yield at the ninth month. Fertilizer application of $400 \mathrm{~kg} / \mathrm{ha}$ gave higher tuber and garri yields at ninth and twelfth month of harvest. Application of $200 \mathrm{~kg} / \mathrm{ha}$ of NPK fertilizer gave significantly higher tuber and garri yields at the sixth month of harvest. Split method of fertilizer application gave significantly higher tuber yield but non-significant higher garri yield at the sixth month of harvest. Split - split fertilizer application gave non-significant higher tuber and garri yields at 12 MAP. There was reduction in tuber and garri yields in the late planting when compared to the early planting.

\section{Interaction effects}

The interaction of variety, rate and mode of fertilizer application showed that TMS 980505 gave significantly $(p<0.05)$ higher number of leaves at 2 and 4 months after planting when the fertilizer was applied split - split (Table 6). The result was statistically similar to TMS 011368 at single and split dose of fertilizer application. The variety also had non-significant higher number of branches, stem girth and canopy diameter at 4, 8 and $12 \mathrm{MAP}$, respectively. The variety TMS 980505 that received $600 \mathrm{~kg} / \mathrm{ha}$ rate of fertilizer application gave significantly $(\mathrm{p}<0.05)$ higher 
112

tuber yield of $27.89 \mathrm{t} / \mathrm{ha}$ and $50.1 \mathrm{t} / \mathrm{ha}$ at the sixth and twelfth months of harvest, respectively (Table 7). It was statistically similar to TMS 0510 that gave 45.6 t/ ha at 600 $\mathrm{kg} / \mathrm{ha}$ of NPK at the twelfth month after planting. The variety TMS 0510 gave significantly higher tuber yield of

$36.67 \mathrm{t} / \mathrm{ha}$ at $200 \mathrm{~kg} / \mathrm{ha}$ of NPK that was applied singly at 6 MAP (Table 8). At 9 and 12 MAP, TMS 980505 gave higher tuber yield of $37 \mathrm{t} / \mathrm{ha}$ and $60 \mathrm{t} / \mathrm{ha}$ at $600 \mathrm{~kg}$ rate that was applied by split and split - split application, respectively.

Table 5. Harvest index and garri yield of the early season planted cassava varieties as influenced by rates and mode of NPK fertilizer application

\begin{tabular}{|c|c|c|c|c|c|c|c|c|c|}
\hline \multirow{3}{*}{$\begin{array}{c}\text { Treatments } \\
\text { Varieties }\end{array}$} & \multicolumn{3}{|c|}{ Tuber yield ( $\mathrm{t}$ )/ha } & \multicolumn{3}{|c|}{ Peel wt $(t) /$ ha } & \multicolumn{3}{|c|}{ Garri yield $(\mathrm{t}) / \mathrm{ha}$} \\
\hline & \multicolumn{9}{|c|}{ Months after planting } \\
\hline & 6 & 9 & 12 & 6 & 9 & 12 & 6 & 9 & 12 \\
\hline \multicolumn{10}{|c|}{ Early season } \\
\hline TMS 011368 & 19.83 & 18.74 & 30.20 & 7.94 & 5.68 & 8.08 & 3.79 & 4.24 & 6.62 \\
\hline TME 419 & 19.17 & 20.58 & 26.70 & 8.94 & 6.25 & 6.81 & 3.79 & 5.44 & 7.31 \\
\hline TMS 980505 & 24.06 & 22.43 & 39.80 & 8.86 & 5.42 & 9.36 & 4.72 & 4.94 & 9.68 \\
\hline TMS 0510 & 21.28 & 22.50 & 31.70 & 6.97 & 5.79 & 7.12 & 4.29 & 5.83 & 8.33 \\
\hline $\operatorname{LSD}(\mathrm{p}<0.05)$ & 3.17 & NS & 5.62 & 1.37 & NS & 1.44 & 0.66 & 1.78 & 1.40 \\
\hline \multicolumn{10}{|l|}{ Rates } \\
\hline 0 & 16.25 & 14.10 & 19.30 & 5.83 & 4.18 & 4.19 & 2.90 & 3.46 & 4.35 \\
\hline 200 & 24.69 & 21.43 & 31.00 & 9.44 & 5.79 & 8.71 & 5.15 & 5.36 & 7.76 \\
\hline 400 & 21.69 & 25.62 & 39.40 & 8.78 & 6.97 & 9.24 & 4.40 & 5.97 & 10.13 \\
\hline 600 & 21.69 & 23.10 & 38.70 & 8.67 & 6.19 & 9.24 & 4.14 & 5.67 & 9.71 \\
\hline LSD $(p<0.05)$ & 3.17 & 3.30 & 5.62 & 1.37 & 1.06 & 1.44 & 0.66 & 1.03 & 1.40 \\
\hline \multicolumn{10}{|l|}{ Modes } \\
\hline Single & 21.94 & 20.05 & 30.00 & 8.46 & 5.69 & 7.66 & 4.03 & 4.66 & 7.14 \\
\hline Split & 22.67 & 21.34 & 32.10 & 8.71 & 5.82 & 8.05 & 4.77 & 5.38 & 8.29 \\
\hline Split-Split & 18.65 & 21.79 & 34.20 & 7.38 & 5.84 & 7.82 & 3.65 & 5.31 & 8.48 \\
\hline $\operatorname{LSD}(\mathrm{p}<0.05)$ & 2.74 & NS & NS & NS & NS & NS & 0.57 & NS & NS \\
\hline \multicolumn{10}{|c|}{ Late season } \\
\hline TMS 011368 & 10.73 & 11.54 & 20.20 & 3.94 & 4.28 & 5.08 & 1.29 & 2.32 & 3.48 \\
\hline TME 419 & 10.17 & 10.58 & 16.70 & 4.14 & 4.23 & 5.31 & 1.53 & 3.04 & 3.41 \\
\hline TMS 980505 & 15.06 & 16.43 & 29.80 & 4.46 & 4.42 & 6.36 & 2.62 & 2.64 & 4.38 \\
\hline TMS 0510 & 12.20 & 13.50 & 21.70 & 3.37 & 4.12 & 5.12 & 2.09 & 2.93 & 3.53 \\
\hline $\operatorname{LSD}(\mathrm{p}<0.05)$ & 2.27 & NS & 3.72 & 1.37 & NS & 1.44 & 0.66 & NS & NS \\
\hline \multicolumn{10}{|l|}{ Rates } \\
\hline 0 & 6.25 & 5.10 & 11.30 & 1.83 & 2.13 & 2.12 & 1.30 & 1.46 & 2.33 \\
\hline 200 & 14.29 & 13.13 & 25.00 & 4.44 & 3.79 & 5.71 & 2.45 & 3.32 & 4.76 \\
\hline 400 & 11.69 & 17.32 & 31.20 & 3.58 & 4.37 & 6.24 & 2.40 & 3.93 & 5.43 \\
\hline 600 & 10.39 & 15.10 & 30.30 & 4.17 & 4.16 & 6.24 & 2.14 & 3.62 & 5.31 \\
\hline $\operatorname{LSD}(\mathrm{p}<0.05)$ & 2.27 & 3.30 & NS & NS & NS & 1.44 & 0.66 & NS & NS \\
\hline \multicolumn{10}{|l|}{ Modes } \\
\hline Single & 11.34 & 14.05 & 20.10 & 3.46 & 3.89 & 5.66 & 2.03 & 2.66 & 3.34 \\
\hline Split & 12.62 & 15.34 & 22.13 & 3.71 & 4.22 & 6.05 & 2.57 & 3.48 & 3.29 \\
\hline Split-Split & 8.25 & 15.09 & 24.20 & 2.18 & 3.84 & 6.82 & 2.45 & 3.31 & 3.45 \\
\hline $\operatorname{LSD}(\mathrm{p}<0.05)$ & 2.26 & NS & NS & NS & NS & NS & NS & NS & NS \\
\hline
\end{tabular}

Table 6. Interaction of varieties $\mathrm{x}$ mode of NPK fertilizer on the number of leaves, branches, canopy diameter and stem girth at different months after planting (MAP)

\begin{tabular}{|c|c|c|c|c|c|c|c|c|c|c|c|c|}
\hline & & 2 & 4 & 2 & 4 & 8 & 10 & 12 & 2 & 4 & 6 & 8 \\
\hline Varieties & Modes & \multicolumn{2}{|c|}{ Number of leaves } & \multicolumn{2}{|c|}{ Number of branches } & \multicolumn{3}{|c|}{ Canopy diameter $(\mathrm{cm})$} & \multicolumn{2}{|c|}{ Stem girth $(\mathrm{cm})$} & & \\
\hline \multirow[t]{3}{*}{ TMS 011368} & Single & 12.23 & 70.90 & 2.00 & 3.62 & 105.50 & 104.4 & 104.80 & 7.21 & 3.90 & 4.53 & 5.72 \\
\hline & Split & 10.09 & 75.00 & 1.77 & 3.51 & 100.20 & 93.10 & 105.20 & 8.19 & 4.38 & 5.22 & 5.80 \\
\hline & $\begin{array}{c}\text { Split - } \\
\text { Split }\end{array}$ & 9.32 & 68.80 & 1.75 & 3.49 & 98.70 & 92.70 & 99.70 & 8.00 & 3.78 & 3.88 & 5.45 \\
\hline \multirow[t]{3}{*}{ TME 419} & Single & 9.48 & 38.30 & 1.61 & 2.75 & 114.20 & 115.10 & 119.30 & 8.77 & 4.43 & 4.49 & 6.09 \\
\hline & Split & 9.69 & 36.90 & 1.69 & 3.08 & 108.00 & 110.20 & 105.80 & 8.41 & 4.55 & 4.68 & 6.16 \\
\hline & $\begin{array}{c}\text { Split - } \\
\text { Split }\end{array}$ & 10.98 & 39.20 & 1.88 & 3.46 & 113.00 & 115.50 & 116.80 & 8.37 & 4.46 & 4.50 & 5.97 \\
\hline \multirow[t]{3}{*}{$\begin{array}{c}\text { TMS } 9805 \\
05\end{array}$} & Single & 13.69 & 72.90 & 2.21 & 3.70 & 117.50 & 114.50 & 121.40 & 4.29 & 5.05 & 4.64 & 5.95 \\
\hline & Split & 14.06 & 64.10 & 2.17 & 2.95 & 111.80 & 117.70 & 119.00 & 4.39 & 5.25 & 6.07 & 5.17 \\
\hline & $\begin{array}{c}\text { Split - } \\
\text { Split }\end{array}$ & 16.58 & 78.40 & 2.15 & 4.26 & 113.90 & 117.90 & 124.60 & 3.77 & 4.61 & 4.48 & 6.23 \\
\hline \multirow[t]{3}{*}{ TMS 0510} & Single & 10.17 & 47.00 & 1.83 & 3.42 & 104.79 & 105.60 & 98.00 & 3.68 & 4.65 & 4.47 & 6.04 \\
\hline & Split & 13.57 & 42.40 & 1.57 & 2.88 & 103.20 & 101.30 & 100.20 & 3.68 & 4.19 & 4.25 & 6.07 \\
\hline & $\begin{array}{c}\text { Split - } \\
\text { Split }\end{array}$ & 11.59 & 43.50 & 1.96 & 2.46 & 94.90 & 96.10 & 103.90 & 3.55 & 4.26 & 4.45 & 5.88 \\
\hline $\operatorname{LSD}(\mathrm{p}<0.05)$ & & 1.47 & 10.41 & NS & NS & NS & NS & NS & NS & NS & NS & NS \\
\hline
\end{tabular}


Table 7. Interaction of varieties $x$ rate of NPK fertilizer application on cassava tuber yield at 6,9 and 12 MAP

\begin{tabular}{|c|c|c|c|c|}
\hline \multirow{2}{*}{ Varieties } & \multirow{2}{*}{ Rates } & \multicolumn{3}{|c|}{ Months after planting (MAP) } \\
\hline & & 6 & 9 & 12 \\
\hline \multirow[t]{4}{*}{ TMS 011368} & 0 & 18.56 & 10.94 & 27.40 \\
\hline & 200 & 20.56 & 19.44 & 24.60 \\
\hline & 400 & 19.33 & 25.89 & 40.30 \\
\hline & 600 & 19.56 & 18.67 & 28.70 \\
\hline \multirow{4}{*}{ TME 419} & 0 & 12.11 & 13.00 & 11.90 \\
\hline & 200 & 23.44 & 18.89 & 26.40 \\
\hline & 400 & 22.11 & 26.56 & 37.80 \\
\hline & 600 & 19.00 & 23.89 & 30.60 \\
\hline \multirow{4}{*}{ TMS 980505} & 0 & 19.44 & 18.06 & 19.20 \\
\hline & 200 & 27.33 & 25.39 & 42.20 \\
\hline & 400 & 21.56 & 24.39 & 47.80 \\
\hline & 600 & 27.89 & 21.89 & 50.10 \\
\hline \multirow{4}{*}{ TMS 0510} & 0 & 12.56 & 14.39 & 18.70 \\
\hline & 200 & 27.44 & 22.00 & 30.90 \\
\hline & 400 & 23.78 & 25.67 & 31.60 \\
\hline & 600 & 21.33 & 27.94 & 35.60 \\
\hline $\operatorname{LSD}(\mathrm{p}<0.05)$ & & 6.33 & NS & 5.67 \\
\hline
\end{tabular}

= non - significant

Table 8. Interaction of variety, rate and mode of NPK fertilizer on cassava tuber yield

\begin{tabular}{|c|c|c|c|c|c|}
\hline \multirow{2}{*}{ Varieties } & \multirow{2}{*}{ Rates } & \multirow{2}{*}{ Modes } & \multicolumn{3}{|c|}{ Months after planting (MAP) } \\
\hline & & & 6 & 9 & 12 \\
\hline \multirow[t]{12}{*}{ TMS 011368} & 0 & Single & 13.33 & 14.50 & 28.70 \\
\hline & & Split & 30.33 & 11.00 & 22.00 \\
\hline & & Split-Split & 19.00 & 7.33 & 31.70 \\
\hline & 200 & Single & 22.67 & 14.67 & 30.70 \\
\hline & & Split & 16.33 & 23.00 & 35.30 \\
\hline & & Split-Split & 22.67 & 20.67 & 31.70 \\
\hline & 400 & Single & 18.67 & 19.00 & 31.00 \\
\hline & & Split & 21.33 & 30.33 & 46.70 \\
\hline & & Split-Split & 18.00 & 22.33 & 43.30 \\
\hline & 600 & Single & 27.67 & 18.67 & 31.30 \\
\hline & & Split & 18.00 & 21.33 & 37.70 \\
\hline & & Split-Split & 10.00 & 16.00 & 17.00 \\
\hline \multirow[t]{12}{*}{ TME 419} & 0 & Single & 12.33 & 12.67 & 12.00 \\
\hline & & Split & 12.00 & 9.67 & 9.70 \\
\hline & & Split-Split & 12.00 & 16.67 & 14.00 \\
\hline & 200 & Single & 22.00 & 17.33 & 22.30 \\
\hline & & Split & 32.33 & 17.00 & 23.70 \\
\hline & & Split-Split & 16.00 & 22.33 & 33.20 \\
\hline & 400 & Single & 18.33 & 20.00 & 31.00 \\
\hline & & Split & 30.00 & 30.33 & 38.30 \\
\hline & & Split-Split & 18.00 & 29.33 & 37.20 \\
\hline & 600 & Single & 20.33 & 20.00 & 33.00 \\
\hline & & Split & 19.00 & 30.33 & 28.30 \\
\hline & & Split-Split & 17.67 & 21.33 & 30.30 \\
\hline \multirow[t]{12}{*}{ TMS 980505} & 0 & Single & 24.33 & 18.17 & 20.30 \\
\hline & & Split & 17.00 & 20.00 & 18.30 \\
\hline & & Split-Split & 17.00 & 16.00 & 19.00 \\
\hline & 200 & Single & 32.67 & 21.00 & 28.30 \\
\hline & & Split & 25.67 & 21.00 & 54.30 \\
\hline & & Split-Split & 23.67 & 34.17 & 44.00 \\
\hline & 400 & Single & 21.00 & 19.00 & 37.30 \\
\hline & & Split & 25.00 & 27.17 & 53.00 \\
\hline & & Split-Split & 18.67 & 27.00 & 53.00 \\
\hline & 600 & Single & 18.00 & 34.33 & 54.70 \\
\hline & & Split & 31.33 & 37.00 & 40.00 \\
\hline & & Split-Split & 34.33 & 34.33 & 60.00 \\
\hline \multirow[t]{11}{*}{ TMS 0510} & 0 & Single & 15.00 & 15.83 & 14.00 \\
\hline & & Split & 14.00 & 12.67 & 17.00 \\
\hline & & Split-Split & 8.67 & 14.67 & 25.00 \\
\hline & 200 & Single & 36.67 & 22.33 & 34.70 \\
\hline & & Split & 25.67 & 16.00 & 28.70 \\
\hline & & Split-Split & 20.00 & 27.67 & 29.30 \\
\hline & 400 & Single & 27.00 & 25.33 & 30.70 \\
\hline & & Split & 19.67 & 20.33 & 28.00 \\
\hline & & Split-Split & 24.67 & 31.33 & 36.20 \\
\hline & 600 & Single & 21.00 & 28.00 & 25.00 \\
\hline & & Split & 25.00 & 28.33 & 52.70 \\
\hline \multirow[t]{2}{*}{$\operatorname{LSD}(p<0.05)$} & & Split-Split & 18.00 & 27.50 & 49.00 \\
\hline & & & 10.97 & NS & 19.48 \\
\hline
\end{tabular}


114

\section{Discussion}

The early season planted cassava took place in May and there were high rainfall in June and July, which supported its rapid early growth and canopy cover. The late season crop which was established in July had low rainfall at the beginning of the crop, but a heavy rainfall in September which was at extreme (stress) and may suggest the basis for low growth and slow canopy cover. Early growth indices in cassava have been reported to support high tuber yield in cassava (Akoroda et al., 2001). El-shakarwy et al. (1998) had also reported that early and mid-season stress significantly reduce top and root biomass than late and terminal stress which occurred during tuber maturity in cassava.

The interpreted physico-chemical properties of soil as contained in Table 1 revealed that the soil of the early season has loamy sand texture and was highly acidic, while the late season site had sandy loam texture and was highly acidic. The soil textural classes of both sites suggest very good support for optimum growth and yield of cassava. Sandy loam of late seasons site has been described as the best for cassava growth. The late season site identified to posse's moderate and high levels of organic carbon and organic matter when compared to the early season site had a reduced or lower yield. This was as a result of reduced effective growth period before dry season started, which was unfavourable for the plant. Early drought observed at late season planting resulted in reduction in tuber yield.

Early season planting that showed higher survival count in all the varieties suggested better growth conditions at early season when compared to the later season with lower survival counts after three weeks of planting. The variety, TMS 980505 that was consistently higher than 70\% in both seasons indicate higher survival rate from rottening and termite attack that predominates in lowering survivors in the late and early seasons respectively. Such a result implies that this genotype could be selected for late season planting

Higher number of leaves and branches at four MAP observed at the early season indicated that the plant growth rate had a favourable environmental condition at the earlier season when compared to late season. El-sharkawy et al. (1998) reported the severe stress effects of dry season on vegetative growth of late season cassava. This suggested that stress at vegetative stage caused reduction in yield of cassava for vegetative growth and eventual tuber yield. Rate of leaf formation in the varieties was related to branch number and rate of leaf retention. The variety TMS 980505 had consistently higher number of branches and leaves in both seasons suggested that the rate of leaf formation in the varieties was related to the number of branches. The report agreed with Irikura et al. (1979) that early branching increase leaf formation rate. Also, Okogbenin (1999) noted that cultivars with high branching characteristics produce more leaves than non-branching. According to Okogun et al. (1999) an increase in number of branches per plant is important to expose the cassava leaves to sunlight for photosynthesis and increased translocation for higher photosynthate accumulation which in turn improves the yield.

The variety TMS 980505 that gave significantly $(\mathrm{p}<$ 0.05 ) higher canopy diameter at the 10 and 12 months after planting and a non- significant higher diameter at 8 month. This indicated that the variety exhibited a full canopy closure. Such variety has the advantage of weed suppression and erosion control because of the vegetation cover. Pellet $e t$ al. (1977) had noted that once a complete ground cover is reached, cassava shed out weeds. Aneke et al. (2010) reported that the application of fertilizer in cassava production ensures that the canopy closes up within approximately three MAP giving potential for weed suppression. Canopy closure has also, been suggested to help to reduce water runoff and consequently reducing soil erosion (Zhang et al., 1998).

The high level of reduction in tuber and garri yield in the late season planting when compared to the early season suggested that the optimum vegetative and tuberization growth period in the late seasons cropping fell into the stress periods of November to March when there was no rainfall. The non-full expression of the leaves and canopy diameter in the late season resulted in lower tuber yield. This is most probable as Lebot (2009) has shown that leaf size and tubers develop simultaneously, also, that increased canopy increases assimilate produced and partitioned between growth and tuberization. The higher tuber and garri yields in TMS 980505 might be as a result of higher canopy cover which increased assimilates produced in the variety for growth and tubers development. The $400 \mathrm{~kg} \mathrm{NPK} / \mathrm{ha}$ that gave significantly higher tuber yield at the ninth and twelfth month is more economical for adoption for production. The non-statistical difference in tuber yield between single and split fertilizer application across the three months of tuber harvest suggested the resilience of the varieties in the utilization of available fertilizer nutrients in their growth and tuberization.

Split-split application of fertilizer to the cassava varieties gave significantly higher tuber yield across some of the varieties used for study at the twelfth month, though it is statistically similar to split application. The result suggested that cassava requires gradual application of fertilizer most probably because of long gestation period of about 12 months. However, the cost of labour should be taken into consideration in the choice of split or split-split for tuber yield compensation from each method. At nine MAP, the non-significantly higher tuber yield at $400 \mathrm{~kg} / \mathrm{ha}$ of fertilizer rate applied split as against the tuber yield at $600 \mathrm{~kg} / \mathrm{ha}$ that was applied split-split suggested that $400 \mathrm{~kg} / \mathrm{ha}$ rate is more adequate and economical for higher tuber yield in the varieties. Similar result of higher tuber yield at $400 \mathrm{~kg} / \mathrm{ha}$ as against $600 \mathrm{~kg} / \mathrm{ha}$ at the 12 month indicated that $400 \mathrm{~kg} / \mathrm{ha}$ rate of fertilizer is suitable for production of the varieties in Nsukka environment because it is more economical and gave high yield. The relatively lower yield from $600 \mathrm{~kg} / \mathrm{ha}$ might be as result of luxuriant growth of the plants at the expense of tuber formation and development. Many authors have reported luxuriant vegetative growth in cassava against tuber formation because of excess nitrogen and potassium levels (Rao et al., 1986; Onwueme and Charles, 1994; Wilson and Ovid, 1994).

The high level of interaction of the cassava varieties with the rates and modes of fertilizers application suggested that the varieties behaved differently to the rates and modes of fertilizers application. At $6 \mathrm{MAP}$, in most cases, the single fertilizer application across the levels gave higher tuber and 
garri yields when compared with the split and split-split. However, single dose application lost its nutrients to leaching over time of growth resulting in lower tuber and garri yields at 9 and 12 MAP. The split - split application of $600 \mathrm{~kg} / \mathrm{ha}$ fertilizer that gave non-significant higher tuber yield of $60 \mathrm{t} / \mathrm{ha}$ when compared with split-split application of $400 \mathrm{~kg} / \mathrm{ha}$ suggested relatively higher fertilizer utilization in the vegetative and tuber bulking and eventual yields. The higher but no significant differences in the yields of 600 $\mathrm{kg} / \mathrm{ha}$ rate may not pay for the cost of extra fertilizer when compared with $400 \mathrm{~kg} /$ ha fertilizer rate. Hence, the lower fertilizer rate of $400 \mathrm{~kg} / \mathrm{ha}$ that gave high yield over the two seasons could be recommended based on the native nutrients of the research site. The variety TMS 980505 also responded in a better way to the rates and modes of fertilizer application as it gave mostly higher tuber yield across the rates and modes of fertilizer application. TME 419 characterised as early ( 6 months) duration cassava did not perform better than other varieties at $6 \mathrm{MAP}$ and did not show rotten tubers at $12 \mathrm{MAP}$ in the seasons in Nsukka environment. Hence, the variety could be grown for 12 MAP harvest as is applicable in some varieties available in Nsukka derived savannah agro-ecology.

\section{Conclusions}

The present study showed that TMS 980505 had significantly high growth parameters and high tuber yield so it could be adopted for production in Nsukka environment. The $400 \mathrm{~kg} / \mathrm{ha}$ rate of fertilizer gave similar growth and tuber yields when compared with $600 \mathrm{~kg} / \mathrm{ha}$ rate and should be adopted because of lower cost. Split application of fertilizer though statistically similar to split -split application had the high growth and tuber yield and should be adopted because it is more efficient economically to the farmers as it will avoid extra labour cost of the fertilizer application.

\section{References}

Aba A (2010). Nigeria's quest for food security. Peoples Daily. Retrieved 2010 March 16, from http/lndu.Nigeriadailynews.com/templates/ defaultaspx. A25979 \& template.

Adeniji OT, Odo PE, Ibrahim B (2011). Genetic relationship and selection indices for cassava root yield in Adamawa State, Nigeria. African Journal of Agricultural Research 6(13):2931-2934.

Akoroda MO, Dixon AGO, Okechukwu RU (2001). Relating early growth traits to root productivity for cassava breeding schemes. Proceedings of the 7th Triennial Symposium of the Rep. of International Society for Tropical Root Crops. Africa Branch held in Benin 11-17 October 1998. Compiled by Akoroda MO and Ngeve J $\mathrm{M}$.

Ande OT (2011). Soil suitability evaluation and management for cassava production in the derived savanna area of south western Nigeria. International Journal of Soil Science 6(2):142-149.

Anneke MF, Tittonell PA, Giller KE (2010). Towards understanding factors that govern fertilizer response in cassava: lessons from East Africa. Nutrient Cycling in Agroecosystemes 86(1):133-151.

Anyaegbunam HW, Okoye BC, Asumugha GN, Ogbonna MC, Madu
TU, Nwakor N, Ejechi ME (2010). Labour productivity among small holder Cassava farmers in South East agro ecological zone, Nigeria. Africa Journal of Agricultural Research 5(21):2882-2885.

Balagopalan C (2002). Cassava utilization in food, feed and industry. In: Hillocks RJ, Thresh JM, Bellotti AC (Eds). Cassava biology, production and utilization. New York: CABI Publishing pp 301-318.

Black CA (1975). Methods of soil analysis. Agronomy No 9, Part 2. American Society of Agronomy. Madison, Wisconsin.

Bray RH, Kurtz LT (1945). Determination of total, organic and available form of phosphorus in soils. Soil Science 59(1):39-46.

Bremner JM (1996). Methods of soil analysis: Chemical methods(No. 631.417/S736V.3).SSSA.

Burns A, Gleadow R, CliffJ,Zacarias A, Cavagnoro T (2010). Cassava: The drought, war and famine crop in a changing world. Sustainability 2:3572-3607.

Cardoso CEL, Souza J da S (1999). Aspectos agro-economicos da cultura da mandioca: potencialidades e limitacoes. [Agro-economic aspects of cassava culture: potentialities and limitations]. Cruz das Almas: Embrapa Mandiocae Fruticultura. Documentos 86.

Carter SE, Fresco IO, Jones PG, Faribarn JN (1992). An atlas cassava in Africa: historical, agroecological and demographic aspects of crop distribution. Center International de Agricultura Tropical Publication No. 206, Cali, Colombia.

El-sharkawy MA (1993). Drought-tolerant cassava for Africa, Asia and Latin America. Bioscience 43:441-451.

El-sharkawy MA, Cadadis LF, Tafur SM, Caicedo JA (1998). Genotypic differences in productivity by prolonged water stress. Acta Agronomica 48(1-2):9-22.

Ene LSO (1992). Prospects for processing and utilization of roots and tuber crops. In: National Root Crops Promotion of Root-Based Industries pp $7-11$.

Ezedinma CN Nkang, Simon I (2006). Price transmission and market integration: A test of the central market hypothesis of geographical markets for cassava products in Nigeria. Global Journal of Pure and Applied Sciences 15(3-4).

Gee GW, Bauders A (1986). Particle size analysis. In: Khite A(Ed). Methods of Soil Analysis, Part 1 (2nd ed). Agronomy Monograph 9, ASA and SSA, Madison, WL.

Godfrey AI, Ezekiel UU,Donatus FU (2012). Selection criteria for stem and tuber yield in cassava (Manihot esculenta Crantz). Journal of American Science 8:1120-1124.

Gomez KA, Gomez AA (1984). Statistical procedure for agricultural research.John Wiley and Sons, USA pp 104115.

Howeler RH (1991). Long term effects of cassava cultivation on productivity. Field Crop Research 26(1):1-18.

IITA (International Institute of Tropical Agriculture) (1990). Cassava in tropical agriculture: A practical manual. International Root Crop-based Industries (1989) pp 46.

Irikura Y, CockJH, Kawano K(1979). The physiological basis of genotypetemperature interactions in cassava. Field Crops Research 2:227-239.

Lahai MT, Ekanayaka IJ (2009). Accumulation and distribution of dry matter in relation to root yield of cassava under a fluctuating water in inland valley ecology. African Journal of Biotechnology 8:4895-4905. 
116

Lebot V (2009). Tropical root and tuber crops: cassava, sweet potato, yams and aroids. CABI Publication.

Leon J (1997). Origin, evolution and early dispersal of root and tuber crops. In: Proceedings of the 4th Symposium of the International Society for Tropical Root Crops. International Development Research Centre, Ottawa, Canada pp 20-36.

Mbagwu JSC (1992). Improving the productivity of a degraded utisol in Nigeria using organic and inorganic amendments. Part 1: chemical properties and maize yield. Bioresource Technology 42:149-154.

Nelson DW, LE Sommers (1996). Total carbon, organic carbon and organic matter. Methods of soil analysis, Part 3 - Chemical methods pp 961-1010.

Njoku DN, Afuape SO, Ebeniro CN (2010). Growth and yield of cassava as influenced by grain cowpea population density in southern Nigeria. African Journal for Agricultural Research 5:2778-2781.

Nweke FI (1994). Processing potential for cassava production growth in Africa.

Nweke FI, Spencer D, Lynam JK (2002). The cassava transformation: Africa's best kept secret. Michigan State University Press.

Odurukwe SO, Ikeorgu JEG (1994). Effects of fertilizer and time of introduction of cassava in yam/maize/cassava intercrop on component yields. In: Symposium on Tropical Root Crops in a Developing Economy 380:72-77.

Ogundari K, Ojo SO (2007). An examination of technical, economic and allocative efficiency of small farms: The case study of cassava farmers in Osun State of Nigeria. Journal of Central European Agriculture $7(3): 423-432$.

Okeke JE (1996). Effect of spatial arrangement and relative time of planting on intercropped maize and cassava. Tropical Science 36(1):6-10.

Okeke JE (1998). Cassava production in Nigeria. National Root Crops Research Institute, Umuahia, AbiaState.

Okogbenin E, Ekanayaka IJ, Porto MCM (1999).Effect of planting material and soil moisture on cassava performance in the semi-arid Sudan savanna belt of Nigeria. African Crop Science Journal 7:21-33.
Okogun JA, Sanginga N, Adeola EO (1999). Soil fertility maintenance and strategies for cassava production in West and Central Africa. IITA, Ibadan, Nigeria.

Onwueme IC, Charles WB (1994). Tropical root and tuber crops: production, perspective and future prospects. Food and Agriculture Organization of the United Nations.

Pellet DM, El Sharkawy MA (1997). Cassava varietal response to fertilization: growth dynamics and implications for cropping sustainability. Experimental Agriculture 33(3):353-365.

Rao DM, Rao MS, Reddy KVS (1986). Nitrogen and potassium requirements of cassava. The Journal of Research APAU 14(2):157161.

Sims IT (1996). Lime requirement. Pp 491-515. In: Sparks DL (Ed). Methods of soil analysis. Part 3. Chemical Methods. SSSA and ASA Madison, WI.

Udealor A (2002). Studies on growth, yield, organic matter turnover and soil nutrient changes in cassava (Manihot esculenta Crantz) vegetable cowpea (Vigna unguilata (L) walp) mixtures. PhD Thesis, University of Nigeria, Department ofCropScience, Nsukka, Nigeria.

Walkley A, Black IA (1934). An examination of Degtjareff method for determining soil organic matter and proposed modification of chronic and titration method. Soil Science 73:29-38.

Yakasi MT (2010). Economic contribution of cassava production (a case study of Kuje area council federal capital territory, Abuja, Nigeria). BayeroJournal of Pure and Applied Sciences 3(1):215-219.

Zhang W, Lingh X, Kaimian L (1998). Cassava breeding, agronomy and farmer participatory research in Asia. Proceedings of the fifth regional workshop held at CATAS, Danzhou, Hainan, China, 3-8 Nov 1996. Centro Internacional de Agricultura Tropical, Regional Cassava Program for Asia. 